\title{
4D Soft Material Systems
}

\author{
Giulia Grassi ${ }^{1(\otimes)}$, Bjorn Sparrman ${ }^{2}$, Ingrid Paoletti ${ }^{1}$, and Skylar Tibbits ${ }^{2}$ \\ 1 Material Balance Research, ABC Department, Politecnico di Milano, via Ponzio 31, \\ 20133 Milan, Italy \\ giulia.grassi@polimi.it \\ 2 Self-Assembly Lab, Massachusetts Institute of Technology, 265 Massachusetts Avenue, \\ Cambridge, MA 02139, USA
}

\begin{abstract}
This work introduces multi-material liquid printing as an enabling technology for designing programmed shape-shifting silicones. The goal of this research is to provide a readily available, scalable and customized approach at producing responsive $4 \mathrm{D}$ printed structures for a wide range of applications. Hence, the methodology allows customization at each step of the procedure by intervening either on the material composition and/or on the design and fabrication strategies for the production of responsive components. A significant endeavour is initiated to develop and engineer two different material systems that enable shape-shifting: silicone-ethanol composites and polyvinyl siloxane swelling rubbers. The printed samples successfully comply with the expected swelling behaviour through a variety of printed test patterns.
\end{abstract}

Keywords: 4D printing $\cdot$ Responsive material systems $\cdot$ Shape-shifting silicones

\section{The Dance of Agencies}

Polish scientist and philosopher Ludwik Fleck (Fleck 1979), introduced a vision of research practice in which the active part of the researcher deals with setting up the material assemblage, and the passive part consists in observing what material will do and how they will perform. Those phases are repeated by the researcher in a loop where the steps of human passivity can be seen as material activity, in a "dance of human and non-human agency in which activity and passivity on both sides are reciprocally intertwined."

Material agency denotes the possibility that things can act on their own which contributes to a broader challenging of the boundaries between ontological categories (Van Oyen 2018). In recent years we have experienced a fertile generation of architecture focused on material systems, such interplay of material innovation, advanced material processes and emerging fabrication technologies is increasingly expanding our understanding of material practice (Perez 2011). A material is nowadays perceived as the active generator of design (Grassi et al. 2021), made possible through techniques like 4D printing which allow designers to fully exploit material engineering and fabrication techniques to produce responsive material systems. 
In this research, 4D printing design strategies, such as shape-shifting multi-material bi-layers, are coupled with Rapid Liquid Printing, a printing technique which entails physically drawing three-dimensionally in a gel suspension, with the aim of investigating soft responsive material systems. Contemporary explorations on the aesthetics of soft spaces and architectures have been exploring silicone as a soft, transparent and flexible rubber, e.g. for inflatables structures such as "Liquid Printed Pneumatics" (Sparrman et al. 2019). Hence silicone is a valuable material for this investigation, thanks to its elasticity, enabling kinetic morphable shapes. Taking advantage of the inherent material properties by distributing the actuation throughout the surfaces, the self-transforming process eliminates the need for external forces, actuators, or human/robot intervention during the shape-shifting process. Moreover, silicone possess strong bonding characteristics, flexibility (a wide array of shore hardness availability), bio-compatibility, versatility, fire resistance and durability.

\section{4D Rapid Liquid Printing}

Previous research on 4D printing typically relies on the use of high-end multi-material printers (such as Stratasys Connex 500), which employ their own proprietary materials, or custom lab-engineered material properties. As a result, experimentation on active materials is difficult for designers to access and scale towards applications. A novel printing technique called Rapid Liquid Printing (RLP) (Hajash et al. 2017), spatially extrudes two-part liquid materials immersed within a tank of gel, avoiding the need for scaffolding. In this study we demonstrate that, by utilizing such technology coupled with multi-material extrusion, it is possible to print silicone-based responsive material systems that can self-transform.

This project investigates two main research questions:

- Which silicone-based composite materials can be used to achieve a shape-shifting response through Rapid Liquid Printing (RLP)?

- Which design strategies and fabrication procedures are needed to create a responsive material system with RLP?

As shown in Fig. 1, a three-axis grantry-style CNC machine has been equipped with the two-part pneumatic deposition system and a tank of gel which serves as a suspension medium. The pneumatic deposition system consists of two cartridges filled with twopart liquid material (1:1 ratio) that is pushed out by a compressor conveying through a static mixer. Different nozzles can be employed depending on the printing diameter (or line spacing) and printing speed. These factors are influenced by the viscosity of the liquid printing material and its curing time (thus printing time for the overall piece or set of prints). Multi-material Rapid Liquid Printing was achieved by swapping material cartridges throughout the printing process. 

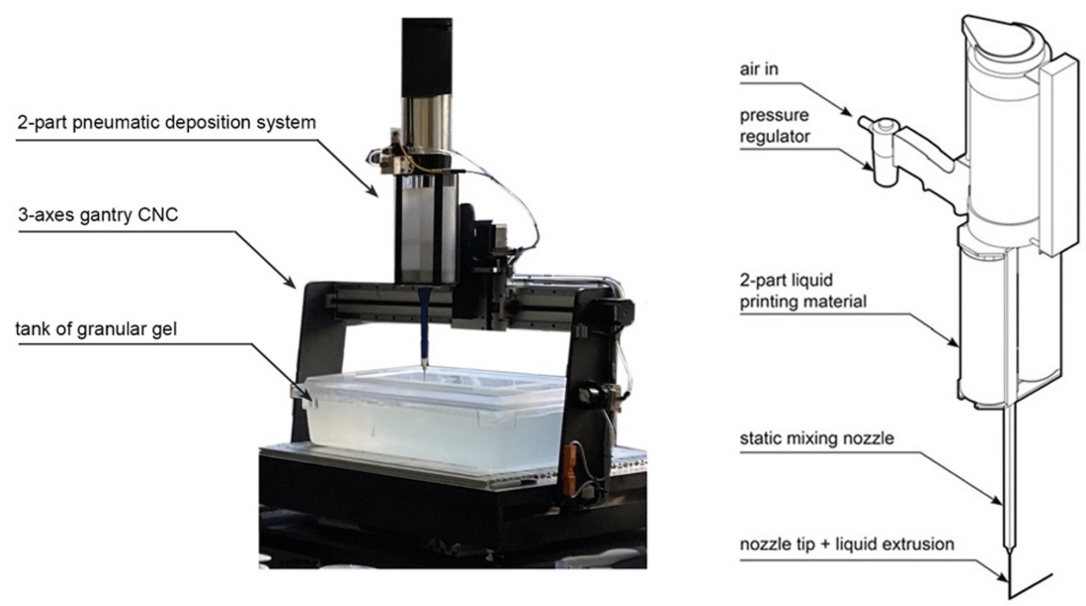

Fig. 1. Machine setup for RLP

\section{Shape-Shifting Silicones}

By exploiting bi-layer compositions and multi-material printing it is possible to mix the silicone matrix with a responsive material coupled with a passive silicone layer. We analyzed the state of the art and possible applications of two material systems with shape-shifting abilities. These have been tested for feasibility, first in terms of desired adaptive behavior as well as machine compatibility. The two material systems are:

- Silicone bilayers of stimuli responsive material systems (active response) tested by mixing a platinum catalyzed silicone with a phase changing material in order to make the composite thermostrictive (ehanol)

- Silicone bilayers as passively activated rubbers (passive response) achieved by printing bi-layers of oil swelling polyvinyl siloxane (PVS) with various oil contents and Young modulus

Hereby we consider as an active transformation when the shape-shifting is triggered by a change in the external environment, whereas a passive transformation is generated by internal forces due to chemical reactions.

\subsection{Ethanol-Based Active Responsive Material System}

The first material system that was tested for printing was a compound of silicone and ethanol. While undergoing phase change from a liquid to gas when reaching the boiling point, ethanol expands. This composite material has been shown to combine high actuation stress and expansion of up to about $900 \%$ (Miriyev et al. 2017). After activation, the component reverses to the previous state once the heat source is removed. Previous studies include projects developed at IAAC (Institute for Advanced Architecture of Catalonia), So.ar (Abasova et al. 2019) and Pneu.flex (Jose et al. 2018). These investigated 
the potential of cast mixtures of silicone and ethanol heated through a coiled Nichrome wire for fabricating responsive skins. Other significant studies have been conducted at Columbia University, at the Creative Machines Lab. They have 3D printed composite materials with the purpose of creating soft actuators (Miriyev et al. 2019) with custom shapes, however in a small scale (the build plate is $406 \times 406 \times 76 \mathrm{~mm}$ ). Moreover, the system doesn't allow for growing in height with the print, because of the liquid nature of silicone, despite UV curing. On the other hand, RLP allows larger scale, due to the bigger size of the tank and tanks to the support given by the gel.

\subsubsection{Material Testing}

For the material system we tested different compositions starting from cast samples. As described in the literature (Miriyev et al. 2017, 2018a, 2018b; Jose et al. 2018), 20\% vol. ethanol in the mixture allows for the optimal expansion rate of the compound. We tested 10, 20 and 30\% weight (slightly higher than \% in vol. because the specific gravity of ethanol is 0.8 ), where $30 \%$ left the specimens wet while not exhibiting an increase in expansion. We cast a few samples of bi-layers made of a passive layer (plain silicone) with an active one, composed of silicone and ethanol, which starts responding to heat at $40{ }^{\circ} \mathrm{C}$. Upon heating, the stress mismatch between the two layers initiates the shapechange. The samples were heated with a heat-gun and the response was approximately within one minute. Two different silicones have been employed as a matrix: Smooth On Sorta Clear and Polytek PlatSil Gel-25. The cast samples with Polytek silicone achieved a more dramatic bending radius because of the lower Shore hardness of the material.

Figure 2 displays a printed bi-layer strip ( $10 \mathrm{~cm}$ length, $3 \mathrm{~cm}$ width) which is actuated through a heat-gun in $1 \mathrm{~min}$ and $40 \mathrm{~s}$. The two layers are printed starting from the lower one and swapping cartridges to change material. In this phase it is crucial to carefully consider the single layer height, thus the two layer distance, in order to print the second layer on top of the first one. To ensure adhesion, when the first layer starts to cure (but is not yet fully cured), the second has to be pushed into the first layer by setting the layer distance lower than the layer height. This approach takes into account the viscosity and the thixotropy of the material and most importantly, the curing time.
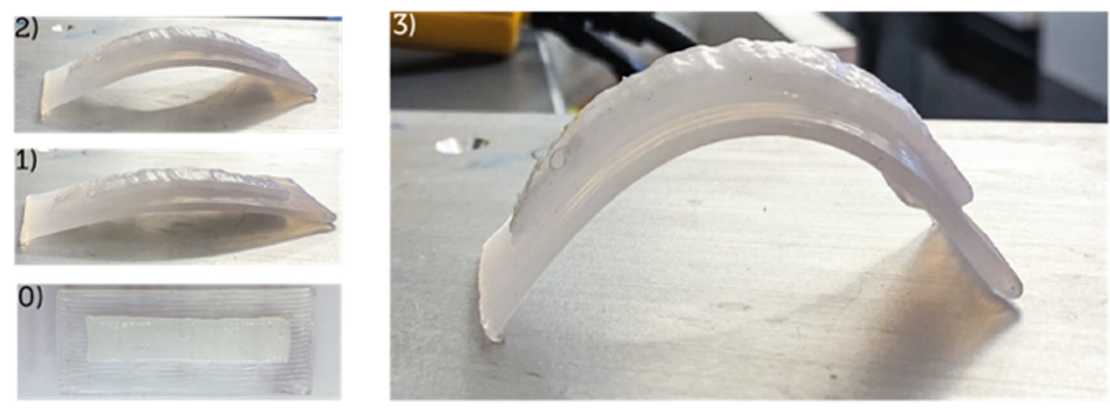

Fig. 2. Printed bi-layer activation, the transition from 0 to 3 took $1 \mathrm{~min}$ and $40 \mathrm{~s}$. 


\subsubsection{Thermo-Responsive Apertures}

By exploiting the successful bending feature of activated bi-layers, more complex and larger geometries have been printed such as the star-like aperture shown in Fig. 3. The sample demonstrates the ability to print interesting kinetic geometries that can be exploited for environmentally adaptive products and structures. The star-like aperture has a diameter of $15 \mathrm{~cm}$ and was printed in less than $5 \mathrm{~min}$. It consists of two layers where the first is plain silicone and the second is a silicone-ethanol mixture. The planar line spacing is $0.75 \mathrm{~mm}$ and the printed path has been optimized ("spiralized") with a script in order to result in a single continuous printed line for each layer. The layer height is $3 \mathrm{~mm}$, hence the distance between the two layers was set $1 \mathrm{~mm}$ apart in order to have a $0.5 \mathrm{~mm}$ of superimposition through pushing downwards to ensure adhesion.

Several tests on the material composition have been performed to obtain the right viscosity, printing time and cure time of the overall objects. Table 1 indicates values of percentage in weight of silicone, ethanol, thickener and retarder for the active layer. The active layer is printed by mixing the material pushed out by the two cartridges, each one filled respectively with part A and part B, where additives are included in the same ratio to both, to obtain the same viscosity.

Table 1. Composition of the silicone-ethanol mixture for the printed sample in Fig. 3

\begin{tabular}{l|l|l|l|l|l}
\hline & Gel 25 partA & Gel 25 partB & Ethanol & Retarder & Thickener \\
\hline \% weight & 38 & 38 & 20 & 1 & 3 \\
\hline
\end{tabular}

This composition has to be customized in respect to the printing strategy adopted, especially with respect to the retarder, which influences the cure time. For instance, if the same print consists of two layers with two different materials, a higher percentage of retarder will allow it to keep the material in the fluid state (not fully cured) while printing the second layer, which will guarantee bonding. We observed that above 5\% retarder resulted in weakened material properties or uncured parts.

The transition of the responsive element is quick at high temperatures (approximately two minutes at $70^{\circ} \mathrm{C}$ ) however, if we imagine such systems to be activated through solar heat we'd have to integrate an additive to render the composite more heat conductive, thus transforming faster. This issue has been addressed by Xia (2020) which used diamond nanoparticle-based thermally conductive filler to improve the actuation speed.

\subsection{PVS-Based Passive Responsive Material System}

Passively actuated adaptive systems represent another possibility within shape-changing systems. Expanding upon the work of Pezzulla et al. (2015), we conducted a series of experiments aimed at testing the swelling capacity of polyvinylsiloxane (PVS) bi-layers. As investigated by Prof. Holmes from the MOSS Lab at Boston University, harnessing anisotropic swelling allows for precise control over the curvature in bilayer structures. 
a)
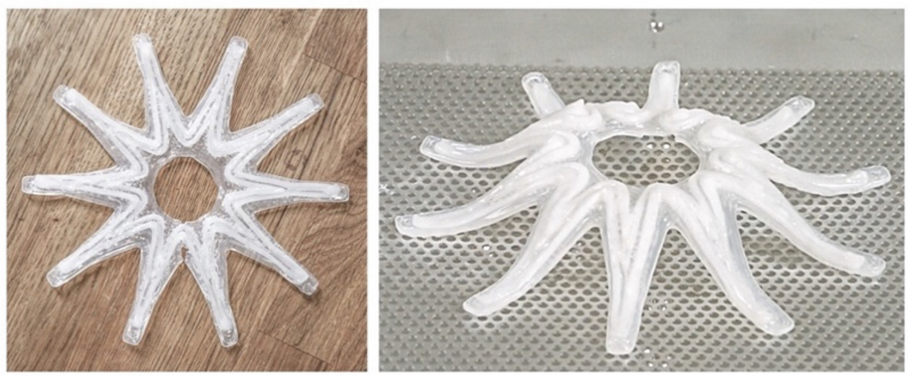

b)

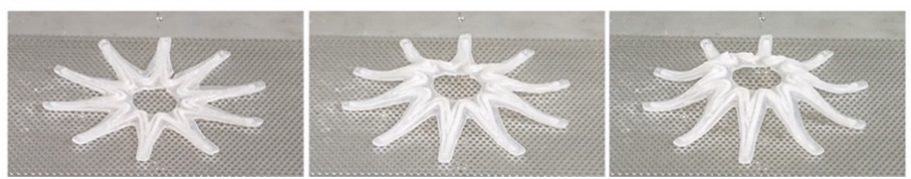

Fig. 3. Star-like shape growing in height upon heating

\subsubsection{Material Testing}

PVS is a two-part silicone mixed in a 1:1 ratio. The tested specimens were cast in bilayers disks with two different silicones (PVS Zhermack Elite Double 32, green, and Zhermack Elite Double 8, pink) in which one layer expands relatively to the other. The differential swelling of the two silicones, with different Young modulus (pink silicone has a lower Shore hardness A, in respect to the green, 8 to 32), is accomplished by the residual polymer chains left in portions of cured elastomers. Consequently, the system exhibits a physical transformation in response to its internal micro behavior and induced stresses. We'll refer to the PVS 32 green as the passive material and to PVS 8, pink, as the active material.

Following the Timoshenko bimetal model we produced casted circular disks of $10 \mathrm{~cm}$ diameter, with different thickness ratios for the two layers. Once the two-part material cured, the samples were removed and tested for their transformation. Their final curvature was affected both by the total thickness as well as the relative thickness of one layer to the other.

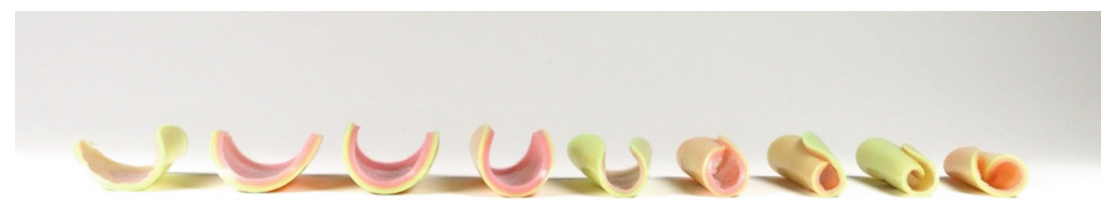

Fig. 4. Tests arranged in order of magnitude of curvature, from left: 14, 11, 10, 13, 18, 0, 17, 15, 12,16

As shown in Fig. 4, the final curvature achieved after curing decreases as the overall thickness of the disks increases. For a fixed quantity of green material, the highest 
curvature has been achieved with the lower quantity of pink. However, the highest curvature has been achieved with a higher quantity of pink (by weight) with respect to the green layer. These tests have shown that the best results can be achieved having a ratio of 2.5 between the green and the pink layer thicknesses. Assuming that the same ratio of 2.5 could apply to the volume too, when printing, the results were achieved by either having different layer heights or by changing the patterns (thus surface area). Viscosity was measured and tuned through the thickener to achieve a range of 800.000-1.000.000 cP to allow printability and to influence the print speed which, for instance, has to faster for a more liquid paste. Furthermore, a retarder was added (1-4\%) in order to tune the cure time according to the design and fabrication needs.

\subsubsection{Shape-Shifting Swelling Rubbers}

Initial printed experiments were conducted with two-layered simple geometries such as rectangles and circles by varying the layers thicknesses and patterns to achieve a gradient of curvatures accordingly to the printed tests. An interesting finding, as shown in Fig. 6 (bottom left), was by taking advantage of the liquid phase of the silicone (increasing the amount of retarder according to the printing time), it was also possible to print a layer of PVS 8 (pink) underneath, on top, or inside the green layer. While the viscosity of PVS 32 was still low, the nozzle could pass through it and print underneath it simplifying the multi-material printing process. Indeed, in order to print a three layer structure made of two materials, instead of swapping the cartridges two times, it was possible to change them only once. Regarding the pattern actuation, the main direction of the material expansion/shrinkage, and the resulting bending orientation, depended on the main direction of the active material. For instance, as shown in Fig. 5 concentric circles would create a positive Gaussian curvature. Other printed objects demonstrated the ability of lattice structures to constrain the deformation of a surface (Fig. 7). While in Fig. 8, the local curvature induced by the swelling, creates a surface change in the regular dotted pattern on the other side of the piece, generating a morphable fur.

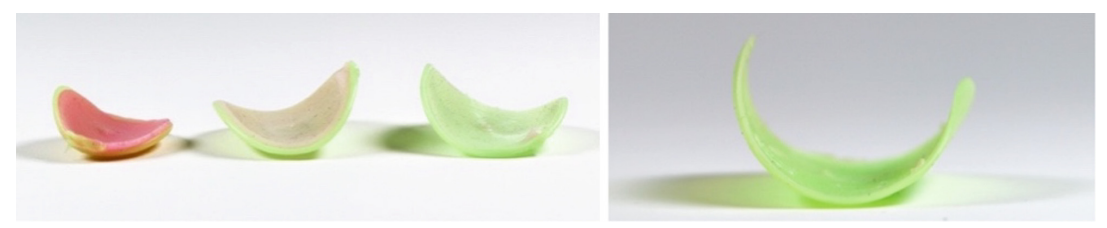

Fig. 5. Left: from left to right - ratio values of the green layer area over the pink are 1,2 and 3. Right: the ratio of the green layer over the pink is 3 and the overall thickness is reduced. 

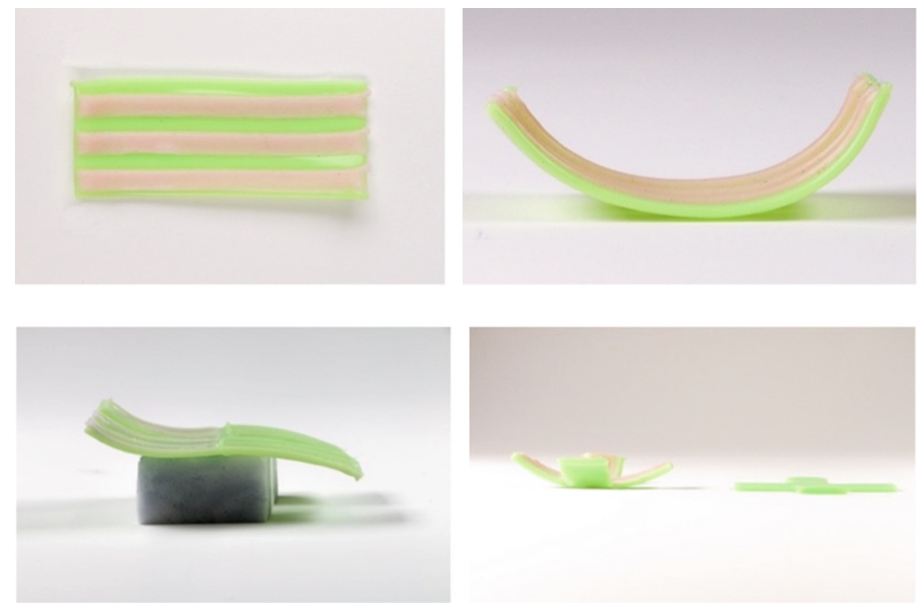

Fig. 6. Printing patterns of the active layer: from one side to two sides stripes and full layer

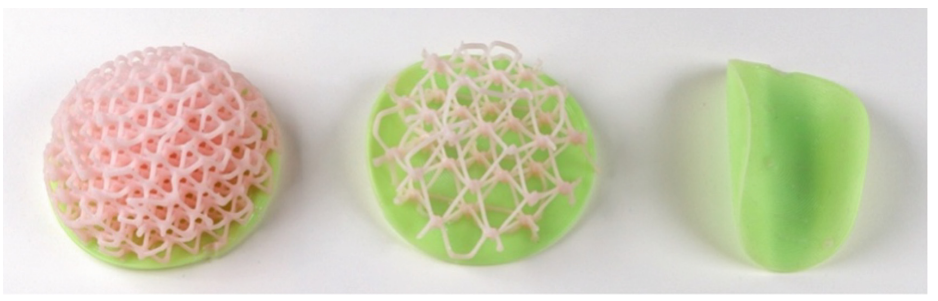

Fig. 7. Lattice structures used to constrain the actuation of a surface

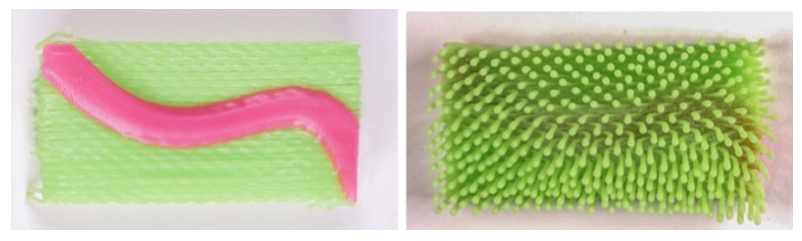

Fig. 8. Morphable fur

\section{Discussion and Conclusions}

Throughout our experiments silicone exhibited extreme flexibility in terms of material testing enabling optimization both of the mix design and of the fabrication process. As a matter of fact, the methodology applied in this research allows for customization at each step of the procedure by intervening either on the material composition and/or on the design and fabrication strategies for the production of active components. This expands the current domain of 4D printing research which has been constrained to proprietary materials and machines or complex processes in laboratory environments that are difficult to scale. Our approach exploits off-the-shelf two-part silicones that are 
widely available and have a wide range of material applications, combined with a novel form of printing to create complex and precise structural transformations. Applications of tunable 4D printed silicones can include active hybrid material systems as sensorialresponsive environments (Ahlquist 2019), soft shape-change tangible interfaces (Ou et al. 2016), and wearables emotional interfaces (Farahi 2018).

Both the silicone-ethanol mixture and PVS bi-layers have proven to be feasible materials for RLP. The main challenges for silicone-ethanol were related to silicone porosity and ethanol volatility, which jeopardize the durability of the material system. On the other side, PVS bi-layers tests, are not reversible once actuated. Therefore, future works can focus on improving the durability of the material systems herein presented and designing more complex structures.

Finally, 4D Soft Material Systems have proven to enable a wide array of kinetic design with an easy-available material such as silicone.

\section{References}

Abasova, A., Sundaram, D., Tarun Kovuru, S.S.: S O.A R - IAAC Blog [WWW Document] (2019). http://www.iaacblog.com/programs/s-o-r/. Accessed 31 Dec 2020

Ahlquist, S.: Expanding the systemic agency of a material system. In: ACADIA 19: Ubiquity and Autonomy; Proceedings of the 39th Annual Conference of the Association for Computer Aided Design in Architecture, pp. 630-641 (2019)

Farahi, B.: Heart of the matter. In: ACADIA 18: Recalibration. On Imprecision and Infidelity. Proceedings of the 38th Annual Conference of the Association for Computer Aided Design in Architecture, pp. 206-215 (2018)

Fleck, L.: Genesis and Development of a Scientific Fact. University of Chicago Press, Chicago (1979)

Grassi, G., Sparrman, B.E., Tibbits, S.: Material agency and 4D printing. In: Paoletti, I., Nastri, M. (eds.) Material Balance. SAST, pp. 53-63. Springer, Cham (2021). https://doi.org/10.1007/ 978-3-030-54081-4_5

Hajash, K., Sparrman, B., Guberan, C., Laucks, J., Tibbits, S.: Large-scale rapid liquid printing. 3D print. Addit. Manuf. 4, 123-132 (2017). https://doi.org/10.1089/3dp.2017.0037

Jose, K., Tabassum, N., Du, Y.: PNEU.FLEX - IAAC Blog [WWW Document] (2018). http:// www.iaacblog.com/programs/pneu-flex/. Accessed 31 Dec 2020

Miriyev, A., Caires, G., Lipson, H.: Functional properties of silicone/ethanol soft-actuator composites. Mater. Des. 145, 232-242 (2018a). https://doi.org/10.1016/j.matdes.2018.02.057

Miriyev, A., Stack, K., Lipson, H.: Soft material for soft actuators. Nat. Commun. (2017). https:// doi.org/10.1038/s41467-017-00685-3

Miriyev, A., Trujillo, C., Caires, G., Lipson, H.: Rejuvenation of soft material-actuator. MRS Commun. 8(2), 556-561 (2018b). https://doi.org/10.1557/mrc.2018.30

Miriyev, A., Xia, B., Joseph, J.C., Lipson, H.: Additive manufacturing of silicone composites for soft actuation. 3D print. Addit. Manuf. 6. (2019). https://doi.org/10.1089/3dp.2019.0116

Ou, J., Dublon, G., Cheng, C.Y., Heibeck, F., Willis, K., Ishii, H.: Cilllia - 3D printed micropillar structures for surface texture, actuation and sensing. In: Conference on Human Factors in Computing Systems - Proceedings. Association for Computing Machinery, New York, NY, USA, pp. 5753-5764 (2016). https://doi.org/10.1145/2858036.2858257

Perez, S.: Towards an ecology of making. In: Borden, G.P., Meredith, M. (eds.) Matter: Material Processes in Architectural Production. Routledge, Abingdon (2011) 
Pezzulla, M., Shillig, S.A., Nardinocchi, P., Holmes, D.P.: Morphing of geometric composites via residual swelling. Soft Matter 11, 5812-5820 (2015). https://doi.org/10.1039/C5SM00863H

Sparrman, B., Kernizan, S., Laucks, J., Tibbits, S., Guberan, C.: Liquid printed pneumatics. In: ACM SIGGRAPH 2019 Emerging Technologies, SIGGRAPH 2019. Association for Computing Machinery, Inc. (2019). https://doi.org/10.1145/3305367.3340318

Van Oyen, A.: Material agency. In: The Encyclopedia of Archaeological Sciences, pp. 1-5. Wiley, Hoboken (2018). https://doi.org/10.1002/9781119188230.saseas0363

$\mathrm{Xia}$, B., et al.: Improving the actuation speed and multi-cyclic actuation characteristics of silicone/ethanol soft actuators. Actuators 9, 62 (2020). https://doi.org/10.3390/ACT9030062

Open Access. This chapter is licensed under the terms of the Creative Commons Attribution 4.0 International License (http://creativecommons.org/licenses/by/4.0/), which permits use, sharing, adaptation, distribution and reproduction in any medium or format, as long as you give appropriate credit to the original author(s) and the source, provide a link to the Creative Commons license and indicate if changes were made.

The images or other third party material in this chapter are included in the chapter's Creative Commons license, unless indicated otherwise in a credit line to the material. If material is not included in the chapter's Creative Commons license and your intended use is not permitted by statutory regulation or exceeds the permitted use, you will need to obtain permission directly from the copyright holder.

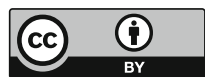

